\title{
Interacting Ricci Dark Energy with Logarithmic Correction
}

\author{
Antonio Pasqua ${ }^{1} *$, A. Khodam-Mohammadi ${ }^{2,3}$ \\ $\dagger$, Mubasher Jamil ${ }^{4,5} \ddagger$ and R. Myrzakulov 6 \\ 1 Department of Physics, University of Trieste, \\ Via Valerio, 234127 Trieste, Italy \\ 2 Physics Department, Faculty of Science, \\ Bu-Ali Sina University, Hamedan 65178, Iran \\ 3 Department of Physics, Faculty of Science, \\ Shiraz University, Shiraz 71454, Iran \\ 4 Center for Advanced Mathematics and Physics (CAMP), \\ National University of Sciences and Technology (NUST), H-12, Islamabad, Pakistan \\ 5 Eurasian International Center for Theoretical Physics, \\ Eurasian National University, Astana 010008, Kazakhstan \\ 6 Department of Physics, California State University, Fresno, CA 93740 USA
}

\begin{abstract}
Motivated by the holographic principle, it has been suggested that the dark energy density may be inversely proportional to the area $A$ of the event horizon of the universe. However, such a model would have a causality problem. In this work, we consider the entropy-corrected version of the holographic dark energy model in the non-flat FRW universe and we propose to replace the future event horizon area with the inverse of the Ricci scalar curvature. We obtain the equation of state (EoS) parameter $\omega_{\Lambda}$, the deceleration parameter $q$ and $\Omega_{D}^{\prime}$ in the presence of interaction between Dark Energy (DE) and Dark Matter (DM). Moreover, we reconstruct the potential and the dynamics of the tachyon, K-essence, dilaton and quintessence scalar field models according to the evolutionary behavior of the interacting entropy-corrected holographic dark energy model.
\end{abstract}

\footnotetext{
* Email:toto.pasqua@gmail.com

$\dagger$ Email:khodam@basu.ac.ir

¥Email:mjamil@camp.nust.edu.pk

$\S$ Email:rmyrzakulov@csufresno.edu
} 


\section{INTRODUCTION}

It is popularly believed among astrophysicists and cosmologists that our universe is experiencing an accelerated expansion. The evidence of the accelerated expansion of the universe is proved by numerous and complementary cosmological observations, like the Supernovae Ia (SNIa) [Perlmutter et al. 1999, Astier et al. 2006], the Cosmic Microwave Background (CMB) anisotropies, observed mainly by WMAP (Wilkinson Microwave Anisotropy Probe) |Bennett et al. 2003, Spergel 2003|, the Large Scale Structure (LSS) Tegmark et al. 2004, Abazajian et al. 2004, Abazajian et al. 2005] and X-ray Allen et al. 2004] experiments.

In the framework of standard Freidmann-Robertson-Walker (FRW) cosmology, a missing energy component with negative pressure (called Dark Energy (DE)) is the source of this expansion. Careful analysis of cosmological observations, in particular of WMAP data Spergel 2003, Bennett et al. 2003, Peiris et al. 2003] indicates that the two-thirds of the total energy of the universe is been occupied by the DE whereas DM occupies almost the rest (the baryonic matter representing only a few percent of the total).

The nature of DE is still unknown, and scientists have proposed many candidates in order to describe it (see [Padmanabhan 2003, Peebles \& Ratra 2003, Copeland et al. 2006] and references therein for good reviews).

The time-independent cosmological constant, $\Lambda$, as vacuum energy density, with equation of state (EoS) parameter $\omega=-1$ is the earliest, most famous and simplest theoretical candidate for DE.

Cosmologists know that the cosmological constant suffers from two well-known difficulties: the fine-tuning and the cosmic coincidence problems [Copeland et al. 2006]. The former asks why the vacuum energy density is so small (an order of $10^{-123}$ smaller than what

we observe) [Weinberg 1989] and the latter says why vacuum energy and DM are nearly equal today (which is an incredible coincidence if there are no internal connections between them).

The alternative candidates for DE problem are the dynamical DE scenarios with no longer constant but time-varying EoS, $\omega$. According to some analysis on the SNe Ia observational data, it has been shown that the time-varying DE models give a better fit compared with a cosmological constant. There are two different categories for dynamical DE scenarios: 
(i) scalar fields, including quintessence Wetterich 1988, Ratra \& Peebles 1988], Kessence [Chiba et al 2000, Armendariz-Picon et al. 2000, Armendariz-Picon et al. 2001], phantoms [Caldwell 2002, Noiiri \& Odintsov 2003, Noiiri \& Odintsov 2003], tachyon Sen 2002b, Padmanabhan 2002, Padmanabhan \& Choudhurv 2002], dilaton Gasperini et al. 2002, Piazza \& Tsuijkawa 2004, Arkani-Hamed et al. 2004], quintom [Elizalde et al. 2004, Nojiri et al. 2005, Anisimov 2005] and so forth, and (ii) interacting DE models, including Chaplygin gas models Kamenshchik et al. 2001, Setare 2007a, Bento et al. 2002], braneworld models [Deffavet et al. 2002, Sahni \& Shtanov 2003], holographic DE (HDE) [Cohen et al. 1999, Hořava \& Minic 2000, Setare 2007d, Setare 2007f, Setare 2006, Setare 2007e, Setare 2007c, Setare 2007b] and agegraphic DE (ADE) models [Cai 2007, Wei \& Cai 2008]. For a good review about the problem of DE, including a survey of some theoretical models, see [Li et al. 2011].

An important advance in the studies of black hole theory and string theory is the suggestion of the so called holographic principle. According to the holographic principle, the number of degrees of freedom of a physical system should be finite and should scale with its bounding area rather than with its volume [t'Hooft 1993] and it should be constrained by an infrared cut-off [Cohen et al. 1999]. The holographic DE (HDE), based on the holographic principle proposed by [Fischler \& Susskind 1998], is one of the most interesting DE candidates and it has been widely studied in literature Huang \& Li 2004, Hsu 2004, Wang et al. 2005b, Guberina et.al 2005, Guberina et al. 2006, Gong 2004, Jamil \& Farooq 2010a, Jamil et al. 2011, Sadiadi \& Jamil 2011, Shevkhi \& Jamil 2011, Shevkhi \& Jamil 2011, Shevkhi 2010, Elizalde et al. 2005, Setare 2007c, Setare \& Jamil 2010c, $\quad$ Setare \& Jamil 2011, $\quad$ Setare \& Jamil 2010a, Karami et al. 2011, $\quad$ Faroog et al. 2010b, Shevkhi et al. 2010, Jamil et al. 2010, Jamil et al. 2009a, Karami et al. 2010, Sheykhi 2009, Setare \& Shafei 2006, Setare 2007d, Setare \& Vagenas 2008, Setare 2006, Setare 2007a, Zhang \& Wu 2005, Zhang 2006, Zhang \& Wu 2007, Enqvist et al. 2005, Shen et al. 2005, Jamil et al. 2009b]. HDE models have also been constrained and tested by various astronomical observations Zhang \& Wu 2005, Zhang \& Wu 2007, Huang \& Li 2004, Enqvist et al. 2005, Wang \& Xu 2010, Micheletti 2010, Zhang 2009, Feng et al. 2005, Kao et al. 2005, Shen et al. 2005] as well as by the anthropic principle [Huang \& Li 2005].

Applying the holographic principle to cosmology, the upper bound of the entropy contained 
in the universe can be obtained [Fischler \& Susskind 1998]. Following this line, [Li 2004] suggested the following constraint on its energy density:

$$
\rho_{\Lambda} \leq 3 c^{2} M_{p}^{2} L^{-2}
$$

where $c$ is a numerical constant, $L$ denotes the IR cut-off radius, $M_{p}=(8 \pi G)^{-1 / 2}$ is the reduced Planck mass ( $G$ represents the gravitational constant) and the equality sign holds only when the holographic bound is saturated. Obviously, in the derivation of HDE, the black hole entropy $S_{B H}$ plays an important role. As it is well known, $S_{B H}=A /(4 G)$, where $A \approx L^{2}$ is the area of horizon and $G$ is the gravitational constant. However, in literature, this entropyarea relation can be modified to Baneriee \& Maihi 2008, Baneriee \& Ranian Maihi 2008, Baneriee \& Modak 2009, Majhi 2009, Jamil \& Farooq 2010b, Wei et al. 2010, Easson et al. 2010, Mohseni Sadjadi \& Jamil 2010, Jamil \& Sheykhi 2011]:

$$
S_{B H}=\frac{A}{4 G}+\tilde{\alpha} \log \left(\frac{A}{4 G}\right)+\tilde{\beta},
$$

where $\tilde{\alpha}$ and $\tilde{\beta}$ are dimensionless constants of order of unity. These corrections can appear in the black hole entropy in Loop Quantum Gravity (LQG). They can also be due to thermal equilibrium fluctuation, quantum fluctuation, or mass and charge fluctuations. The quantum corrections provided to the entropy-area relationship leads to the currvature correction in the Einstein-Hilbert action and viceversa ZZhu \& Ren 2009, Cai et al. 2009, Nojiri \& Odintsov 2001]. Using the corrected entropy-area relation (21), the energy density of the entropy-corrected HDE (ECHDE) can be written as [Wei 2009]:

$$
\rho_{\Lambda}=3 \alpha M_{p}^{2} L^{-2}+\gamma_{1} L^{-4} \log \left(M_{p}^{2} L^{2}\right)+\gamma_{2} L^{-4}
$$

where $\gamma_{1}$ and $\gamma_{2}$ are dimensionless constants of order unity. In the limiting case $\gamma_{1}=\gamma_{2}=0$, Eq. (3) yields the well-known HDE density.

The first term on Eq. (3) corresponds to the usual holographic energy density. The second and the third terms are due to entropy corrections: since they can be comparable to the first term only when $L$ is very small, the corrections given by them make sense only at the early evolutionary stage of the universe. When the universe becomes large, Eq. (33) reduce to that of the ordinary HDE.

Inspired by the HDE models, in this paper we propose to consider another possibility: the 
IR cut-off radius $L$ is given by the average radius of Ricci scalar curvature, $R^{-1 / 2}$, so that we have the DE density $\rho_{\Lambda} \propto R$. We remember that the Ricci scalar can be written as:

$$
R=6\left(\dot{H}+2 H^{2}+\frac{k}{a(t)^{2}}\right),
$$

where $H=\dot{a}(t) / a(t)$ is the Hubble parameter, $\dot{H}$ is the derivative of the Hubble parameter with respect to the cosmic time $t, a(t)$ is a dimensionless scale factor and $k$ is the curvature parameter which can assume the values $-1,0,+1$ which yield, respectively, an open, a flat or a closed FRW universe.

Substituting $L$ with $R^{-1 / 2}$, we can write the energy density of Ricci ECHDE (R-ECHDE) as:

$$
\rho_{\Lambda}=3 \alpha M_{p}^{2} R+\gamma_{1} R^{2} \log \left(M_{p}^{2} / R\right)+\gamma_{2} R^{2},
$$

where $\alpha, \gamma_{1}$ and $\gamma_{2}$ are three constants, $M_{p}=(8 \pi G)^{-1 / 2}$ is the modified Planck mass ( $G$ is the gravitational constant). many authors applied the entropy correction terms in an interacting/non-interacting and flat/non-flat universe with modified IR-cutoff (for example see Khodam-Mohammadi \& Malekjani 2011a, Khodam-Mohammadi 2011, Khodam-Mohammadi \& Malekjani 2011b]).

This paper is organized as follows. In Section 2, we describe the physical contest we are working in and we derive the EoS parameter $\omega_{\Lambda}$, the deceleration parameter $q$ and $\Omega_{D}^{\prime}$ for our model in a non-flat universe. In Section 3, we establish a correspondence between our model and the tachyon, K-essence, dilaton and quintessence fields. In Section 4 we write the Conclusions of this paper.

\section{INTERACTING MODEL IN A NON-FLAT UNIVERSE}

Observational evidence suggest that our universe is not perfectly flat but it has a small positive curvature, which implies a closed universe. The tendency of a closed universe is shown in cosmological (in particular CMB) experiments Bennett et al. 2003, Spergel 2003, Tegmark et al. 2004, Seliak et al. 2006, Spergel 2007, Seievers 2003, Netterfield 2002, Benoit et al. 2003, Benoit et al. 2003]. Moreover, the measurements of the cubic correction to the luminosity-distance relation of Supernova measure-

ments reveal a closed universe [Caldwell \& Kamionkowski 2004, Wang et al. 2005a]. For the 
above reasons, we prefer to consider a non-flat universe.

Within the framework of the standard Friedmann-Robertson-Walker (FRW) cosmology, the line element for non-flat universe is given by:

$$
\begin{aligned}
& d s^{2}= \\
& -d t^{2}+a^{2}(t)\left(\frac{d r^{2}}{1-k r^{2}}+r^{2}\left(d \theta^{2}+\sin ^{2} \theta d \varphi^{2}\right)\right)
\end{aligned}
$$

The corresponding Friedmann equation takes the form:

$$
H^{2}+\frac{k}{a^{2}}=\frac{1}{3 M_{p}^{2}}\left(\rho_{\Lambda}+\rho_{m}\right),
$$

where $\rho_{\Lambda}$ and $\rho_{m}$ are the energy densities of DE and DM, respectively.

We also define the fractional energy densities for matter, curvature and DE, respectively, as:

$$
\begin{aligned}
& \Omega_{m}=\frac{\rho_{m}}{\rho_{c r}}=\frac{\rho_{m}}{3 M_{p}^{2} H^{2}}, \\
& \Omega_{k}=\frac{\rho_{k}}{\rho_{c r}}=\frac{k}{H^{2} a^{2}}, \\
& \Omega_{\Lambda}=\frac{\rho_{\Lambda}}{\rho_{c r}}=\frac{\rho_{\Lambda}}{3 M_{p}^{2} H^{2}},
\end{aligned}
$$

where $\rho_{c r}=3 M_{p}^{2} H^{2}$ represents the critical density. The parameter $\Omega_{k}$ represents the contribution to the total density from the spatial curvature. Recent observations support a closed universe with a small positive curvature $\Omega_{k} \cong 0.02$ [Spergel 2007].

Using Eqs. (8), (9) and (10) it is possible to write the Friedmann Eq. (7) in the following form:

$$
\Omega_{m}+\Omega_{\Lambda}=1+\Omega_{k}
$$

In order to preserve the Bianchi identity or local energy-momentum conservation law, i.e. $\nabla_{\mu} T^{\mu \nu}=0$, the total energy density $\rho_{t o t}=\rho_{\Lambda}+\rho_{m}$ must satisfy the following equation:

$$
\dot{\rho}_{t o t}+3 H(1+\omega) \rho_{t o t}=0
$$

where $\omega=p_{t o t} / \rho_{t o t}$ is the total EoS. By assuming an interaction between DE and DM, the two energy densities $\rho_{\Lambda}$ and $\rho_{m}$ are conserved separately and the conservation equations take the following form:

$$
\begin{aligned}
& \dot{\rho}_{\Lambda}+3 H \rho_{\Lambda}\left(1+w_{\Lambda}\right)=-Q, \\
& \dot{\rho}_{m}+3 H \rho_{m}=Q .
\end{aligned}
$$


$Q$ represents the interaction term which can be, in general, an arbitrary function of cosmological parameters, like the Hubble parameter $H$ and energy densities $\rho_{m}$ and $\rho_{\Lambda}$, i.e. $Q\left(H \rho_{m}, H \rho_{\Lambda}\right)$. The simplest choice for $Q$ is:

$$
Q=3 b^{2} H\left(\rho_{m}+\rho_{\Lambda}\right)
$$

with $b^{2}$ a coupling parameter between DM and DE Amendola \& Tocchini-Valentini 2001, Amendola \& Tocchini-Valentini 2002, Setare \& Jamil 2010b, Shevkhi \& Jamil 2011, Farooq et al. 2010a, Jamil \& Farooq 2010a, Jamil \& Saridakis 2010, Zimdahl 2001, Zimdahl 2003, Setare \& Jamil 2010c] although more general interaction terms can be used [Jamil \& Rashid 2008b]. However, since the nature of DM and DE remains unknown, different Lagrangians have been proposed to generate this interaction term. Positive values of $b^{2}$ indicate transition from DE to matter and vice versa for negative values of $b^{2}$. Sometimes $b^{2}$ is taken in the range [0,1] [Zhang \& Zhu 2006]. The case with $b^{2}=0$ represents the non-interacting FRW model while $b^{2}=1$ yields the complete transfer of energy from DE to matter. Recently, it is reported that this interaction is observed in the Abell cluster A586 showing a transition of DE into DM and vice versa [Bertolami et al. 2007, Jamil \& Rashid 2008a]. However the strength of this interaction is not clearly identified [Feng et al. 2007].

Observations of the $\mathrm{CMB}$ and of galactic clusters show that the coupling parameter $b^{2}<0.025$, i.e. a small but positive constant of order of the unity Ichiki \& Keum 2008, Amendola et al. 2007]. A negative coupling parameter is avoided due to violation of thermodynamical laws.

We now want to derive the expression for the EoS parameter $\omega_{\Lambda}$ for our model.

Using (7), the Ricci scalar can be written as:

$$
R=6\left(\dot{H}+H^{2}+\frac{\rho_{m}+\rho_{\Lambda}}{3 M_{p}^{2}}\right) .
$$

From the Friedmann Eq. (7) we obtain that:

$$
\dot{H}=\frac{k}{a^{2}}-\frac{1}{2 M_{p}^{2}}\left(\rho_{m}+\rho_{\Lambda}\left(1+\omega_{\Lambda}\right)\right) .
$$

Adding Eqs. (7) and (17), we obtain:

$$
\dot{H}+H^{2}=\frac{\rho_{m}+\rho_{\Lambda}}{3 M_{p}^{2}}-\frac{1}{2 M_{p}^{2}}\left(\rho_{m}+\rho_{\Lambda}\left(1+\omega_{\Lambda}\right)\right) .
$$


Therefore, the Ricci scalar can be written as:

$$
R=\frac{\rho_{m}+\rho_{\Lambda}}{M_{p}^{2}}-\frac{3 \rho_{\Lambda} \omega_{\Lambda}}{M_{p}^{2}}
$$

From Eq. (19) we can easily derive the expression of the EoS parameter $\omega_{\Lambda}$ :

$$
\omega_{\Lambda}=-\frac{R M_{p}^{2}}{3 \rho_{\Lambda}}+\frac{\rho_{\Lambda}+\rho_{m}}{3 \rho_{\Lambda}}=-\frac{R M_{p}^{2}}{3 \rho_{\Lambda}}+\frac{\Omega_{\Lambda}+\Omega_{m}}{3 \Omega_{\Lambda}} .
$$

Substituting the expression of the energy density given in Eq. (15) and using Eq. (11) we obtain:

$$
\begin{aligned}
\omega_{\Lambda}= & -\frac{M_{p}^{2} / 3}{3 \alpha M_{p}^{2}+\gamma_{1} R \log \left(M_{p}^{2} / R\right)+\gamma_{2} R}+ \\
& \frac{\left(1+\Omega_{k}\right)}{3 \Omega_{\Lambda}} .
\end{aligned}
$$

We now want to derive the expression for the evolution of energy density parameter $\Omega_{\Lambda}$. From Eq. (13), it is possible to obtain the following expression for the EoS parameter $\omega_{\Lambda}$ :

$$
\omega_{\Lambda}=-1-\frac{\dot{\rho}_{\Lambda}}{3 H \rho_{\Lambda}}-\frac{Q}{3 H \rho_{\Lambda}} .
$$

Using the expression of $Q$ given in Eq. (15), the derivative of the DE density $\rho_{\Lambda}$ can be written as:

$$
\dot{\rho}_{\Lambda}=3 H\left[-\rho_{\Lambda}-\left(\rho_{m}+\rho_{\Lambda}\right)\left(b^{2}+\frac{1}{3}\right)+\frac{R M_{p}^{2}}{3}\right] .
$$

Dividing by the critical density $\rho_{c}=3 H^{2} M_{p}^{2}$, Eq. (23) can be written as:

$$
\begin{aligned}
\frac{\dot{\rho}_{\Lambda}}{\rho_{c}} & =\dot{\Omega}_{\Lambda}+2 \Omega_{\Lambda} \frac{\dot{H}}{H} \\
& =3 H\left[-\Omega_{\Lambda}-\left(1+\Omega_{k}\right)\left(b^{2}+\frac{1}{3}\right)+\frac{R}{9 H^{2}}\right] .
\end{aligned}
$$

From Eq. (4), we can see that the term $\frac{R}{9 H^{2}}$ is equivalent to:

$$
\frac{R}{9 H^{2}}=\frac{2}{3}\left(\frac{\dot{H}}{H^{2}}+2+\Omega_{k}\right) .
$$

From Eq. (24) and substituting Eq. (25), it is possible to obtain the derivative of $\Omega_{\Lambda}$ with respect to the cosmic time $t$ :

$$
\begin{aligned}
\dot{\Omega}_{\Lambda}= & 2 \frac{\dot{H}}{H}\left(1-\Omega_{\Lambda}\right)+ \\
& 3 H\left[-\Omega_{\Lambda}-\left(1+\Omega_{k}\right)\left(b^{2}-\frac{1}{3}\right)+\frac{2}{3}\right] .
\end{aligned}
$$


Since $\Omega_{\Lambda}^{\prime}=\frac{d \Omega_{\Lambda}}{d x}=\frac{1}{H} \dot{\Omega}_{\Lambda}$ (where $\left.x=\ln a\right)$, we derive:

$$
\begin{aligned}
H \Omega_{\Lambda}^{\prime}= & 2 H^{\prime}\left(1-\Omega_{\Lambda}\right)+ \\
& 3 H\left[-\Omega_{\Lambda}-\left(1+\Omega_{k}\right)\left(b^{2}-\frac{1}{3}\right)+\frac{2}{3}\right],
\end{aligned}
$$

which yields to:

$$
\begin{aligned}
\Omega_{\Lambda}^{\prime}= & \frac{2}{H}\left(1-\Omega_{\Lambda}\right)+ \\
& 3\left[-\Omega_{\Lambda}-\left(1+\Omega_{k}\right)\left(b^{2}-\frac{1}{3}\right)+\frac{2}{3}\right] .
\end{aligned}
$$

In the above Equation we used the fact that:

$$
H^{\prime}=\frac{a^{\prime}}{a}=1
$$

For completeness, we also derive the deceleration parameter $q$ :

$$
q=-\frac{\ddot{a} a}{\dot{a}^{2}}=-1-\frac{\dot{H}}{H^{2}} .
$$

q, combined with the Hubble parameter $H$ and the dimensionless density parameters, form a set of very useful parameters for the description of the astrophysical observations. Taking the derivative respect to the cosmic time $t$ of the Friedmann Eq. (17) and using Eqs. (11), (13) and (14), it is possible to write Eq. (30) as:

$$
q=\frac{1}{2}\left[1+\Omega_{k}+3 \Omega_{\Lambda} \omega_{\Lambda}\right]
$$

Substituting the expression of the EoS parameter $\omega_{\Lambda}$ given in Eq. (21), we obtain that:

$$
q=1-\frac{1}{2} \frac{M_{p}^{2} \Omega_{\Lambda}}{3 \alpha M_{p}^{2}+\gamma_{1} R \log \left(M_{p}^{2} / R\right)+\gamma_{2} R}+\Omega_{k} .
$$

We can derive the important quantities of the R-ECHDE model in the limiting case, for a flat dark dominated universe in HDE model, i.e. when $\gamma_{1}=\gamma_{2}=0, \Omega_{\Lambda}=1$ and $\Omega_{k}=0$. The energy density given in Eq. (5) reduces to:

$$
\rho_{\Lambda}=3 \alpha M_{p}^{2} R
$$

From FRW Eq. (17) we find:

$$
H=\frac{6 \alpha}{12 \alpha-1}\left(\frac{1}{t}\right)
$$




$$
R=\frac{36 \alpha}{(12 \alpha-1)^{2}}\left(\frac{1}{t^{2}}\right)
$$

At last, the EoS parameter $\omega_{\Lambda}$ and deceleration parameter $q$ reduce to:

$$
\begin{gathered}
\omega_{\Lambda}=\frac{1}{3}-\frac{1}{9 \alpha}, \\
q=1-\frac{1}{6 \alpha} .
\end{gathered}
$$

From Eq. (36), we see that in the limiting case, the EoS parameter of DE becomes a constant value in which for $\alpha<1 / 12, \omega_{\Lambda}<-1$, where the phantom divide can be crossed. Since the Ricci scalar diverges at $\alpha=1 / 12$, this value of $\alpha$ can not be taken into account. From Eq. (37), the acceleration is started at $\alpha \leq 1 / 6$ where the quintessence regime is started $\left(\omega_{\Lambda} \leq-1 / 3\right)$.

This case is very similar to power-law expansion of scale factor of [Granda \& Oliveros 2008], in which $a(t)=t^{6 \alpha /(12 \alpha-1)}$.

\section{CORRESPONDENCE BETWEEN R-ECHDE AND SCALAR FIELDS}

In this Section we establish a correspondence between the interacting Ricci scale model and the tachyon, K-essence, dilaton and quintessence scalar field models. The importance of this correspondence is that the scalar field models are an effective description of an underlying theory of DE. Therefore, it is worthwhile to reconstruct the potential and the dynamics of scalar fields according the evolutionary form of Ricci scalar model. For this purpose, first we compare the energy density of Ricci scale model (i.e. Eq. (5) ) with the energy density of corresponding scalar field model. Then, we equate the equations of state of scalar field models with the EoS parameter of Ricci scalar model (i.e. Eq. (21)).

\section{A. Interacting tachyon model}

Recently, huge interest has been devoted to the study of the inflationary model with the help of the tachyon field, since it is believed the tachyon can be assumed as a possible source of DE [Bagla et al. 2003, Shao et al. 2007, Calcagni \& Liddle 2006, Copeland et al. 2005]. The tachyon is an unstable field which can be used in string theory through its role in 
the Dirac-Born-Infeld (DBI) action to describe the D-bran action [Sen 2002a, Sen 1999, Bergshoeff et al. 2000, Klusoň 2000, Kutasov \& Niarchos 2003]. Tachyon might be responsible for cosmological inflation in the early evolutionary stage of the universe, due to tachyon condensation near the top of the effective scalar potential. A rolling tachyon has an interesting EoS whose parameter smoothly interpolates between -1 and 0 . This discovery motivated to take DE as a dynamical quantity, i.e. a variable cosmological constant and model inflation using tachyons. The effective Lagrangian for the tachyon field is given by:

$$
L=-V(\phi) \sqrt{1-g^{\mu \nu} \partial_{\mu} \phi \partial_{\nu} \phi}
$$

where $V(\phi)$ represents the potential of tachyon and $g^{\mu \nu}$ is the metric tensor. The energy density $\rho_{\phi}$ and pressure $p_{\phi}$ for the tachyon field are given, respectively, by:

$$
\begin{aligned}
& \rho_{\phi}=\frac{V(\phi)}{\sqrt{1-\dot{\phi}^{2}}} \\
& p_{\phi}=-V(\phi) \sqrt{1-\dot{\phi}^{2}} .
\end{aligned}
$$

Instead, the EoS parameter of tachyon scalar field is given by:

$$
w_{\phi}=\frac{p_{\phi}}{\rho_{\phi}}=\dot{\phi}^{2}-1
$$

In order to have a real energy density for tachyon field, it is required that $-1<\dot{\phi}<1$. Consequently, from Eq. (41), the EoS parameter of tachyon is constrained to $-1<\omega_{\phi}<0$. Hence, the tachyon field can interpret the accelerated expansion of the universe, but it can not enter the phantom regime, i.e. $\omega_{\Lambda}<-1$.

Comparing Eqs. (5) and (39), we obtain an expression for the potential $V(\phi)$ of the tachyon:

$$
V(\phi)=\rho_{\Lambda} \sqrt{1-\dot{\phi}^{2}}
$$

Instead, equating Eqs. (21) and (41), we obtain an expression for the kinetic energy term $\dot{\phi}^{2}$ :

$$
\begin{aligned}
& \dot{\phi}^{2}=1+\omega_{\Lambda}= \\
& 1-\frac{M_{p}^{2} / 3}{3 \alpha M_{p}^{2}+\gamma_{1} R \log \left(M_{p}^{2} / R\right)+\gamma_{2} R}+\frac{\left(1+\Omega_{k}\right)}{3 \Omega_{\Lambda}} .
\end{aligned}
$$

Using Eqs. (42) and (43), it is possible to write the potential of the tachyon as:

$$
\begin{aligned}
& V(\phi)=\rho_{\Lambda} \sqrt{-\omega_{\Lambda}}= \\
& \rho_{\Lambda} \sqrt{\frac{M_{p}^{2} / 3}{3 \alpha M_{p}^{2}+\gamma_{1} R \log \left(M_{p}^{2} / R\right)+\gamma_{2} R}-\frac{\left(1+\Omega_{k}\right)}{3 \Omega_{\Lambda}}} .
\end{aligned}
$$


We can derive from Eqs. (43) and (44) that the kinetic energy $\dot{\phi}^{2}$ and the potential $V(\phi)$ may exist if it is satisfied the condition:

$$
-1 \leq \omega_{\Lambda} \leq 0
$$

which implies that the phantom divide can not be crossed in a universe with an accelerated expansion.

Using $\dot{\phi}=\phi^{\prime} H$ and Eq. (43), we get:

$$
\begin{aligned}
& \phi^{\prime}=\frac{1}{H} \times \\
& \sqrt{1-\frac{M_{p}^{2} / 3}{3 \alpha M_{p}^{2}+\gamma_{1} R \log \left(M_{p}^{2} / R\right)+\gamma_{2} R}+\frac{\left(1+\Omega_{k}\right)}{3 \Omega_{\Lambda}} .}
\end{aligned}
$$

Then, from Eq. (46), it is possible to obtain the evolutionary form of the tachyon scalar field as:

$$
\begin{aligned}
& \phi(a)-\phi\left(a_{0}\right)=\int_{a_{0}}^{a} \frac{d a}{a H} \times \\
& \sqrt{1-\frac{M_{p}^{2} / 3}{3 \alpha M_{p}^{2}+\gamma_{1} R \log \left(M_{p}^{2} / R\right)+\gamma_{2} R}+\frac{\left(1+\Omega_{k}\right)}{3 \Omega_{\Lambda}}}
\end{aligned}
$$

where $a_{0}$ is the present value of the scale factor. Here, we have established an interacting entropy-corrected holographic tachyon DE model and reconstructed the potential and the dynamics of the tachyon field.

In the limiting case for flat dark dominated universe for $\gamma_{1}=\gamma_{2}=0, \Omega_{\Lambda}=1$ and $\Omega_{k}=0$, the scalar field and potential of the tachyon are, respectively:

$$
\begin{gathered}
\phi(t)=\sqrt{\frac{12 \alpha-1}{9 \alpha}} t, \\
V(\phi)=\frac{4 M_{p}^{2}}{(12 \alpha-1)} \sqrt{\alpha(1-3 \alpha)} \frac{1}{\phi^{2}},
\end{gathered}
$$

which are a result of the power-law expansion. In this correspondence, the scalar field exist provided that $\alpha>1 / 12$, which shows that the phantom divide can not be achieved.

\section{B. Interacting K-essence model}

A model in which the kinetic term of the scalar field appears in the Lagrangian in a non-canonical way is called K-essence model. The idea of the K-essence scalar field was 
motivated from the Born-Infeld action of string theory and it is used to explain the late time acceleration of the universe [Sen 2002, Lambert \& Sachs 2003]. The general scalar field action for the K-essence model as a function of $\phi$ and $\chi=\dot{\phi} / 2$ is given by [Chiba et al 2000, Armendariz-Picon et al. 2000, Armendariz-Picon et al. 2001]:

$$
S=\int d^{4} x \sqrt{-g} p(\phi, \chi)
$$

where the Lagrangian density $p(\phi, \chi)$ corresponds to a pressure density. According to this Lagrangian, the pressure $p(\phi, \chi)$ and the energy density of the field $\phi$ can be written, respectively, as:

$$
\begin{aligned}
& p(\phi, \chi)=f(\phi)\left(-\chi+\chi^{2}\right), \\
& \rho(\phi, \chi)=f(\phi)\left(-\chi+3 \chi^{2}\right) .
\end{aligned}
$$

Hence, the EoS parameter of K-essence scalar field is given by:

$$
\omega_{K}=\frac{p(\phi, \chi)}{\rho(\phi, \chi)}=\frac{\chi-1}{3 \chi-1}
$$

From Eq. (53), one can see the phantom behavior of K-essence scalar field $\left(w_{K}<-1\right)$ when the parameter $\chi$ lies in the interval $1 / 3<\chi<1 / 2$.

In order to consider the K-essence field as a description of the interacting R-ECHDE density, we establish the correspondence between the K-essence EoS parameter, $\omega_{K}$, and the interacting R-ECHDE EoS parameter, $\omega_{\Lambda}$.

The expression for $\chi$ can be found equating Eqs. (21) and (53), obtaining:

$$
\begin{aligned}
& \chi=\frac{w_{\Lambda}-1}{3 w_{\Lambda}-1}= \\
& \frac{-1-\frac{M_{p}^{2} / 3}{3 \alpha M_{p}^{2}+\gamma_{1} R \log \left(M_{p}^{2} / R\right)+\gamma_{2} R}+\frac{\left(1+\Omega_{k}\right)}{3 \Omega_{\Lambda}}}{-1-\frac{M_{p}^{2}}{3 \alpha M_{p}^{2}+\gamma_{1} R \log \left(M_{p}^{2} / R\right)+\gamma_{2} R}+\frac{\left(1+\Omega_{k}\right)}{\Omega_{\Lambda}}} .
\end{aligned}
$$

Moreover, equating Eqs. (5) and (52), we obtain:

$$
f(\phi)=\frac{\rho_{\Lambda}}{\chi(3 \chi-1)} .
$$

Using $\dot{\phi}^{2}=2 \chi$ and $\dot{\phi}=\phi^{\prime} H$, we derive:

$$
\phi^{\prime}=\frac{\sqrt{2}}{H} \sqrt{\frac{-1-\frac{M_{p}^{2} / 3}{3 \alpha M_{p}^{2}+\gamma_{1} R \log \left(M_{p}^{2} / R\right)+\gamma_{2} R}+\frac{\left(1+\Omega_{k}\right)}{3 \Omega_{\Lambda}}}{-1-\frac{M_{p}^{2}}{3 \alpha M_{p}^{2}+\gamma_{1} R \log \left(M_{p}^{2} / R\right)+\gamma_{2} R}+\frac{\left(1+\Omega_{k}\right)}{\Omega_{\Lambda}}}} .
$$


Integrating Eq. (56), we find the evolutionary form of the K-essence scalar field:

$$
\begin{aligned}
& \phi(a)-\phi\left(a_{0}\right)=\sqrt{2} \int_{a_{0}}^{a} \frac{d a}{a H} \times \\
& \sqrt{\frac{-1-\frac{M_{p}^{2} / 3}{3 \alpha M_{p}^{2}+\gamma_{1} R \log \left(M_{p}^{2} / R\right)+\gamma_{2} R}+\frac{\left(1+\Omega_{k}\right)}{3 \Omega_{\Lambda}}}{-1-\frac{M_{p}^{2}}{3 \alpha M_{p}^{2}+\gamma_{1} R \log \left(M_{p}^{2} / R\right)+\gamma_{2} R}+\frac{\left(1+\Omega_{k}\right)}{\Omega_{\Lambda}}}},
\end{aligned}
$$

where $a_{0}$ is the present value of the scale factor.

In the limiting case of $\gamma_{1}=\gamma_{2}=0, \Omega_{\Lambda}=1$ and $\Omega_{k}=0$, in a flat dark dominated universe, the scalar field and potential of K-essence field reduce to:

$$
\begin{aligned}
\phi(t) & =\sqrt{\frac{2(1+6 \alpha)}{3}} t, \\
f(\phi) & =\frac{36 \alpha M_{p}^{2}}{(12 \alpha-1)^{2}} \frac{1}{\phi^{2}},
\end{aligned}
$$

which are a result of power-law expansion. Moreover, we see that our universe may behave in all accelerated regimes (phantom and quintessence), since all values of $\alpha$ are permitted.

\section{Interacting dilaton model}

A dilaton scalar field, originated from the lower-energy limit of string theory [Piazza \& Tsujikawa 2004], can also be assumed as a source of DE.

The process of compactification of the string theory from higher to four dimensions introduces the scalar dilaton field which is coupled to curvature invariants. The coefficient of the kinematic term of the dilaton can be negative in the Einstein frame, which means that the dilaton behaves as a phantom-like scalar field. The pressure (Lagrangian) density and the energy density of the dilaton DE model are given, respectively, by Gasperini et al. 2002, Arkani-Hamed et al. 2004, Elizalde et al. 2008]:

$$
\begin{aligned}
& p_{D}=-\chi+c e^{\lambda \phi} \chi^{2}, \\
& \rho_{D}=-\chi+3 c e^{\lambda \phi} \chi^{2},
\end{aligned}
$$

where $c$ and $\lambda$ are positive constants and $2 \chi=\dot{\phi}^{2}$. The negative coefficient of the kinematic term of the dilaton field in Einstein frame makes a phantom-like behavior for dilaton field. 
The EoS parameter for the dilaton scalar field is given by:

$$
\omega_{D}=\frac{p_{D}}{\rho_{D}}=\frac{-1+c e^{\lambda \phi} \chi}{-1+3 c e^{\lambda \phi} \chi} .
$$

In order to consider the dilaton field as a description of the interacting R-ECHDE density, we establish the correspondence between the dilaton EoS parameter, $w_{D}$, and the EoS parameter $\omega_{\Lambda}$ of the R-ECHDE model. By equating Eqs. (21) and (62), it is possible to find:

$$
\begin{aligned}
& c e^{\lambda \phi} \chi=\frac{\omega_{\Lambda}-1}{3 \omega_{\Lambda}-1}= \\
& \frac{-1-\frac{M_{p}^{2} / 3}{3 \alpha M_{p}^{2}+\gamma_{1} R \log \left(M_{p}^{2} / R\right)+\gamma_{2} R}+\frac{\left(1+\Omega_{k}\right)}{3 \Omega_{\Lambda}}}{-1-\frac{M_{p}^{2}}{3 \alpha M_{p}^{2}+\gamma_{1} R \log \left(M_{p}^{2} / R\right)+\gamma_{2} R}+\frac{\left(1+\Omega_{k}\right)}{\Omega_{\Lambda}}} .
\end{aligned}
$$

Using $\dot{\phi}^{2}=2 \chi$, we can rewrite Eq. (63) as:

$$
\begin{aligned}
& e^{\lambda \phi / 2} \dot{\phi}=\sqrt{\frac{2}{c}} \times \\
& \sqrt{\frac{-1-\frac{M_{p}^{2} / 3}{3 \alpha M_{p}^{2}+\gamma_{1} R \log \left(M_{p}^{2} / R\right)+\gamma_{2} R}+\frac{\left(1+\Omega_{k}\right)}{3 \Omega_{\Lambda}}}{-1-\frac{M_{p}^{2}}{3 \alpha M_{p}^{2}+\gamma_{1} R \log \left(M_{p}^{2} / R\right)+\gamma_{2} R}+\frac{\left(1+\Omega_{k}\right)}{\Omega_{\Lambda}}}} .
\end{aligned}
$$

Integrating Eq. (64) with respect to $a$, we obtain:

$$
\begin{aligned}
& e^{\frac{\lambda \phi(a)}{2}}=e^{\frac{\lambda \phi\left(a_{0}\right)}{2}}+\frac{\lambda}{\sqrt{2 c}} \int_{a_{0}}^{a} \frac{d a}{a H} \times \\
& \sqrt{\frac{-1-\frac{M_{p}^{2} / 3}{3 \alpha M_{p}^{2}+\gamma_{1} R \log \left(M_{p}^{2} / R\right)+\gamma_{2} R}+\frac{\left(1+\Omega_{k}\right)}{3 \Omega_{\Lambda}}}{-1-\frac{M_{p}^{2}}{3 \alpha M_{p}^{2}+\gamma_{1} R \log \left(M_{p}^{2} / R\right)+\gamma_{2} R}+\frac{\left(1+\Omega_{k}\right)}{\Omega_{\Lambda}}}} .
\end{aligned}
$$

The evolutionary form of the dilaton scalar field is written as:

$$
\begin{gathered}
\phi(a)=\frac{2}{\lambda} \ln \left[e^{\frac{\lambda \phi\left(a_{0}\right)}{2}}+\right]+\frac{\lambda}{\sqrt{2 c}} \int_{a_{0}}^{a} \frac{d a}{a H} \times \\
\sqrt{\frac{-1-\frac{M_{p}^{2} / 3}{3 \alpha M_{p}^{2}+\gamma_{1} R \log \left(M_{p}^{2} / R\right)+\gamma_{2} R}+\frac{\left(1+\Omega_{k}\right)}{3 \Omega_{\Lambda}}}{-1-\frac{M_{p}^{2}}{3 \alpha M_{p}^{2}+\gamma_{1} R \log \left(M_{p}^{2} / R\right)+\gamma_{2} R}+\frac{\left(1+\Omega_{k}\right)}{\Omega_{\Lambda}}}} .
\end{gathered}
$$

In the limiting case of $\gamma_{1}=\gamma_{2}=0, \Omega_{\Lambda}=1$ and $\Omega_{k}=0$, in a flat dark dominated universe, the scalar field of dilaton field reduces to the following form:

$$
\phi(t)=\frac{2}{\lambda} \ln \left[\lambda t \sqrt{\frac{1+6 \alpha}{6 c}}\right] .
$$


We see that all values of $\alpha$ are permitted and, therefore, by this correspondence, the universe may behave in phantom and quintessence regime.

\section{Quintessence}

Quintessence is described by an ordinary time-dependent and homogeneous scalar field $\phi$ which is minimally coupled to gravity, but with a particular potential $V(\phi)$ that leads to

the accelerating universe. The action for quintessence is given by [Copeland et al. 2006]:

$$
S=\int d^{4} x \sqrt{-g}\left[-\frac{1}{2} g^{\mu \nu} \partial_{\mu} \phi \partial_{\nu} \phi-V(\phi)\right]
$$

The energy momentum tensor $T_{\mu \nu}$ of the field is derived by varying the action given in Eq. (68) with respect to the metric tensor $g^{\mu \nu}$ :

$$
T_{\mu \nu}=\frac{2}{\sqrt{-g}} \frac{\delta S}{\delta g^{\mu \nu}}
$$

which yields to:

$$
T_{\mu \nu}=\partial_{\mu} \phi \partial_{\nu} \phi-g_{\mu \nu}\left[\frac{1}{2} g^{\alpha \beta} \partial_{\alpha} \phi \partial_{\beta} \phi+V(\phi)\right]
$$

The energy density $\rho_{Q}$ and pressure $p_{Q}$ of the quintessence scalar field $\phi$ are given, respectively, by:

$$
\begin{aligned}
& \rho_{Q}=-T_{0}^{0}=\frac{1}{2} \dot{\phi}^{2}+V(\phi), \\
& p_{Q}=T_{i}^{i}=\frac{1}{2} \dot{\phi}^{2}-V(\phi) .
\end{aligned}
$$

The EoS parameter for the quintessence scalar field is given by:

$$
\omega_{Q}=\frac{p_{Q}}{\rho_{Q}}=\frac{\dot{\phi}^{2}-2 V(\phi)}{\dot{\phi}^{2}+2 V(\phi)} .
$$

We find from Eq. (73) that, when $\omega_{Q}<-1 / 3$, the universe accelerates for $\dot{\phi}^{2}<V(\phi)$.

Here we establish the correspondence between the interacting scenario and the quintessence DE model: equating Eq. (73) with the EoS parameter given in Eq. (21), i.e. $\omega_{Q}=\omega_{\Lambda}$, and equating Eqs. (71) and(5), i.e. $\rho_{Q}=\rho_{\Lambda}$, we obtain:

$$
\begin{aligned}
\dot{\phi}^{2} & =\left(1+\omega_{\Lambda}\right) \rho_{\Lambda}, \\
V(\phi) & =\frac{1}{2}\left(1-\omega_{\Lambda}\right) \rho_{\Lambda} .
\end{aligned}
$$


Substituting Eq. (22) into Eqs. (74) and (75), the kinetic energy term $\dot{\phi}^{2}$ and the quintessence potential energy $V(\phi)$ can be easily found as follow:

$$
\begin{aligned}
\dot{\phi}^{2}= & \rho_{\Lambda}\left(1-\frac{M_{p}^{2} / 3}{3 \alpha M_{p}^{2}+\gamma_{1} R \log \left(M_{p}^{2} / R\right)+\gamma_{2} R}+\right. \\
& \left.\frac{\left(1+\Omega_{k}\right)}{3 \Omega_{\Lambda}}\right), \\
V(\phi)= & \frac{\rho_{\Lambda}}{2}\left(1+\frac{M_{p}^{2} / 3}{3 \alpha M_{p}^{2}+\gamma_{1} R \log \left(M_{p}^{2} / R\right)+\gamma_{2} R}-\right. \\
& \left.-\frac{\left(1+\Omega_{k}\right)}{3 \Omega_{\Lambda}}\right) .
\end{aligned}
$$

From Eq. (76), using $\dot{\phi}=\phi^{\prime} H$, it is possible to obtain the evolutionary form of the quintessence scalar field as:

$$
\begin{aligned}
& \phi(a)-\phi\left(a_{0}\right)=\int_{a_{0}}^{a} \frac{d a}{a}\left\{\sqrt{3 M_{p}^{2} \Omega_{\Lambda}} \times\right. \\
& \left(1-\frac{M_{p}^{2} / 3}{3 \alpha M_{p}^{2}+\gamma_{1} R \log \left(M_{p}^{2} / R\right)+\gamma_{2} R}+\right. \\
& \left.\left.\frac{\left(1+\Omega_{k}\right)}{3 \Omega_{\Lambda}}\right)^{1 / 2}\right\},
\end{aligned}
$$

where $a_{0}$ is the present value of the scale factor. In the limiting case of $\gamma_{1}=\gamma_{2}=0, \Omega_{\Lambda}=1$ and $\Omega_{k}=0$, in a flat dark dominated universe, the scalar field and potential of quintessence reduce to:

$$
\begin{aligned}
\phi(t) & =\frac{6 \alpha M_{p}}{\sqrt{3 \alpha(12 \alpha-1)}} \ln (t) \\
V(\phi) & =\frac{6 \alpha(6 \alpha+1)}{(12 \alpha-1)^{2}} M_{p}^{2} \exp \left[\frac{-\sqrt{3 \alpha(12 \alpha-1)}}{3 \alpha M_{p}} \phi\right] .
\end{aligned}
$$

The potential exists for all values of $\alpha>1 / 12$ (quintessence regime). The potential has also been obtained by power-law expansion of scale factor.

\section{CONCLUSIONS}

In this paper, we considered the entropy-corrected version of the HDE model which is in interaction with DM in the non-flat FRW universe (and with IR cut-off equivalent to the Ricci scalar R). The HDE model is an attempt to probe the nature of DE within the framework of quantum gravity. We considered the logarithmic correction term to the 
energy density of HDE model. The addition of correction terms to the energy density of HDE is motivated from the Loop Quantum Gravity (LQG), which is one of the most promising theories of quantum gravity. Using the expression of this modified energy density, we obtained the EoS parameter, deceleration parameter and evolution of energy density parameter for the interacting R-ECHDE model. We found that for the appropriate model parameters (even in limiting case, $\gamma_{1}=\gamma_{2}=\Omega_{k}=0, \Omega_{\Lambda}=1$ ), the phantom divide may be crossed, $\omega_{\Lambda}<-1$, and the present acceleration expansion $(q<0)$ is achieved where the quintessence regime is started. Moreover, we established a correspondence between the interacting R-ECHDE model and the tachyon, K-essence, dilaton and quintessence scalar field models in the hypothesis of non-flat FRW universe.

These correspondences are important to understand how various candidates of DE are mutually related to each other. The limiting case of flat dark dominated universe without entropy correction were studied in each scalar field and we see that the EoS parameter is constant in this case and we calculate the scalar field and its potential which can be obtained by idea of power-law expansion of scalar field.

In order to make a comparison between our model and another works in LECHDE-scalar field model, we concentrate our attention in a recent article [Amani et al. 2012]. The authors considered a scalar-tensor cosmological model with the non-minimal kinetic coupling terms and discussed its cosmological implications with respect to the entropy corrected holographic dark energy. Our results differ from their results in that their analysis involves two coupling parameters and a cosmological event horizon while ours deal with a Ricci scale and no couplings. Such scalar field models have interesting property of explaining the phantom crossing while the reconstructed scalar potential has interesting physical implications in cosmology.

\section{Acknowledgments}

We are grateful to the referee for valuable comments and suggestions, which have allowed us to improve this paper significantly. Also M. Jamil would like to thank the warm hospitality 
of the Abdus Salam ICTP, Trieste where this work was initiated.

[Abazajian et al. 2004] Abazajian, K. et al., Astron. J. 128, 502 (2004).

[Abazajian et al. 2005] Abazajian, K. et al., Astron. J. 129, 1755 (2005).

[Allen et al. 2004] Allen, S. W. et al., Mon. Not. R. Astron. Soc. 353, 457 (2004).

[Amani et al. 2012] Amani, A. R., Sadeghi, J., Farajollahi, H., Pourali, M.: Canadian Journal of Physics, 90, 61 (2012).

[Amendola et al. 2007] Amendola, L., Campos, G. C., Rosenfeld, R., Phys. Rev. D 75, 083506 (2007).

[Amendola \& Tocchini-Valentini 2002] Amendola, L., Tocchini-Valetini, D., Phys. Rev. D 66, $043528(2002)$.

[Amendola \& Tocchini-Valentini 2001] Amendola, L., Tocchini-Valetini, D., Phys. Rev. D 64, 043509 (2001).

[Anisimov 2005] Anisimov, A., J. Cosmol. Astropart. Phys. 6, 6 (2005).

[Arkani-Hamed et al. 2004] Arkani-Hamed, N., Creminelli, P., Mukohyama, S., Zaldarriaga, M., J. Cosmol. Astropart. Phys. 4, 1 (2004).

[Armendariz-Picon et al. 2001 ] Armendariz-Picon, C., Mukhanov, V., Steinhardt, P. J., Phys. Rev. D 63, 103510 (2001).

[Armendariz-Picon et al. 2000] Armendariz-Picon, C., Mukhanov, V., Steinhardt, P. J., Phys. Rev. Lett. 85, 4438 (2000).

[Astier et al. 2006] Astier, P. et al., Astron. Astrophys. 447, 31 (2006).

[Bagla et al. 2003] Bagla, J. S., Jassal, H. K., Padmanabhan, T., Phys. Rev. D 67, 063504 (2003).

[Banerjee \& Majhi 2008] Banerjee, R., Majhi, B. R., Phys. Lett. B 662, 62 (2008)..

[Banerjee \& Modak 2009] Banerjee, R., Modak, S. K., JHEP. 5, 63 (2009).

[Banerjee \& Ranjan Majhi 2008] Banerjee, R., Ranjan Majhi, B., JHEP. 6, 95 (2008).

[Bennett et al. 2003] Bennett, C. L. et al., Astrophys. J. 148, 1 (2003).

[Benoît et al. 2003] Benoît, A. et al., Astron. Astrophys. 399, L19 (2003).

[Benoît et al. 2003] Benoît, A. et al., Astron. Astrophys. 399, L25 (2003).

[Bento et al. 2002] Bento, M. C., Bertolami, O., Sen, A. A., Phys. Rev. D 66, 043507 (2002).

[Bergshoeff et al. 2000] Bergshoeff, E. A. et al., JHEP 5, 9 (2000).. 
[Bertolami et al. 2007] Bertolami, O., Gil Pedro, F., Le Delliou, M., Phys. Lett. B 654, 165 (2007).

[Cai 2007] Cai, R. G., Phys. Lett. B 657, 228 (2007).

[Cai et al. 2009] Cai, R.-G., Cao, L.-M., Hu, Y.-P., Class. Quant. Grav. 26, 155018 (2009).

[Calcagni \& Liddle 2006] Calcagni, G., Liddle, A. R., Phys. Rev. D 74, 043528 (2006).

[Caldwell 2002] Caldwell, R. R., Phys. Lett. B 545, 23 (2002).

[Caldwell \& Kamionkowski 2004] Caldwell, R. R., Kamionkowski, M., J. Cosmol. Astropart. Phys. 9, 9 (2004).

[Chiba et al 2000] Chiba, T., Okabe, T., Yamaguchi, M., Phys. Rev. D 62, 023511 (2000).

[Cohen et al. 1999] Cohen, A. G., Kaplan, D. B., Nelson, A. E., Phys. Rev. Lett. 82, 4971 (1999).

[Copeland et al. 2006] Copeland, E. J., Sami, M., Tsujikawa, S., Int. J. Mod. Phys. D 15, 1753 (2006).

[Copeland et al. 2005] Copeland, E. J. et al., Phys. Rev. D 71, 043003 (2005).

[Deffayet et al. 2002] Deffayet, C., Dvali, G., Gabadadze, G., Phys. Rev. D 65, 044023 (2002).

[Easson et al. 2010] Easson, D. A., Frampton, P. H., Smoot, G. F.,(arXiv:1003.1528) [hep-th].

[Elizalde et al. 2005] Elizalde, E., Nojiri, S., Odintsov, S. D., Wang, P., Phys. Rev. D 71, 103504 (2005).

[Elizalde et al. 2004] Elizalde, E., Nojiri, S., Odintsov, Phys. Rev. D 70, 043539 (2004).

[Elizalde et al. 2008] Elizalde, E. et al., Euro. Phys. J. C 53, 447 (2008).

[Enqvist et al. 2005] Enqvist, K., Hannestad, S., Sloth, M. S., J. Cosmol. Astropart. Phys. 2, 4 (2005).

[Farooq et al. 2010a] Farooq, M. U., Jamil, M., Rashid, M. A., Int. J. Theor. Phys. 49, 2278 (2010a).

[Farooq et al. 2010b] Farooq, M. U., Jamil, M., Rashid, M. A., Int. J. Theor. Phys. 49, 2334 (2010b).

[Feng et al. 2007] Feng, C., Wang, B., Gong, Y., Su, R.-K., J. Cosmol. Astropart. Phys. 9, 5 (2007).

[Feng et al. 2005] Feng, B., Wang, X., Zhang, X., Physics Letters B 607, 35 (2005).

[Fischler \& Susskind 1998] Fischler, W., Susskind, L., (arXiv:hep-th/9806039).

[Gasperini et al. 2002] Gasperini, M., Piazza, F., Veneziano, G., Phys. Rev. D 65, 023508 (2002).

[Gong 2004] Gong, Y., Phys. Rev. D 70, 064029 (2004).

[Granda \& Oliveros 2008] Granda, L. N., Oliveros, A., Physics Letters B 669, 275 (2008). 
[Guberina et al. 2006] Guberina, B., Horvat, R., Nikolić, H., Physics Letters B 636, 80 (2006).

[Guberina et.al 2005] Guberina, B., Horvat, R., Stefancic, H., J. Cosmol. Astropart. Phys. 5, 1 $(2005)$.

[Hořava \& Minic 2000] Hořava, P., Minic, D., Physical Review Letters 85, 1610 (2000).

[Hsu 2004] Hsu, S. D. H., Physics Letters B 594, 13 (2004).

[Huang \& Li 2005] Huang, Q.G., Li, M., J. Cosmol. Astropart. Phys. 3, 1 (2005).

[Huang \& Li 2004] Huang, Q.G., Li, M., J. Cosmol. Astropart. Phys. 8, 13 (2004).

[Ichiki \& Keum 2008] Ichiki, K., Keum, Y.Y., J. Cosmol. Astropart. Phys. 6, 5 (2008).

[Jamil et al. 2011] Jamil et al., Int. J. Theor. Phys., 579 (2011).

[Jamil et al. 2010] Jamil, M., Sheykhi, A., Farooq, M. U., International Journal of Modern Physics D 19, 1831 (2010).

[Jamil et al. 2009a] Jamil, M., Saridakis, E. N., Setare, M. R., Physics Letters B 679, 172 (2009).

[Jamil \& Saridakis 2010] Jamil, M., Saridakis, E. N., J. Cosmol. Astropart. Phys. 7, 28 (2010).

[Jamil \& Sheykhi 2011] Jamil, M., Sheykhi, A., International Journal of Theoretical Physics 50, 625 (2011).

[Jamil \& Farooq 2010a] Jamil, M., Farooq, M. U., International Journal of Theoretical Physics 49, 42(2010a).

[Jamil \& Farooq 2010b] Jamil, M., Umar Farooq, M., J. Cosmol. Astropart. Phys. 3, 2 (2010b).

[Jamil \& Rashid 2008a] Jamil, M., Rashid, M. A., European Physical Journal C 58, 111 (2008a).

[Jamil \& Rashid 2008b] Jamil, M., Rashid, M. A., Astrophys. J. 56, 429 (2008b).

[Jamil et al. 2009b] Jamil, M., Farooq, M. U., Rashid, M. A., European Physical Journal C 61, $471(2009 b)$.

[Kamenshchik et al. 2001] Kamenshchik, A., Moschella, U., Pasquier, V., Physics Letters B 511 265 (2001).

[Kao et al. 2005] Kao, H.C., Lee, W.L., Lin, F.L., Phys. Rev. D 71, 123518 (2005).

[Karami et al. 2011] Karami, K., Khaledian, M. S., Jamil, M., Phys. Scr. 83, 025901 (2011).

[Karami et al. 2010] Karami, K., Khaledian, M. S., Felegary, F., zarmi, Z., Physics Letters B 686, 216 (2010).

[Khodam-Mohammadi \& Malekjani 2011a] Khodam-Mohammadi, A., Malekjani, M., Commun. Theor. Phys. 55, 942 (2011).

[Khodam-Mohammadi 2011] Khodam-Mohammadi, A., Mod. Phys. Lett. A 26, 2487 (2011). 
[Khodam-Mohammadi \& Malekjani 2011b] Khodam-Mohammadi, A., Malekjani, M., (arXiv:1101.1632) [gr-qc].

[Klusoň 2000] Klusoň, J., Phys. Rev. D 62, 126003 (2000).

[Kutasov \& Niarchos 2003] Kutasov, D., Niarchos, V., Nuclear Physics B 666, 56 (2003).

[Lambert \& Sachs 2003] Lambert, N. D., Sachs, I., Phys. Rev. D 67, 025005 (2003).

[Li 2004] Li, M., Astrophys. J. 603, 1 (2004).

[Li et al. 2011] Li, M., Li, X.-D., Wang, S., Wang, Y., Commun. Theor. Phys. 56, 525 (2011).

[Majhi 2009] Majhi, B. R., Phys. Rev. D 79, 044005 (2009).

[Micheletti 2010] Micheletti, S. M. R., J. Cosmol. Astropart. Phys. 4, 9 (2010).

[Mohseni Sadjadi \& Jamil 2010] Mohseni Sadjadi, H., Jamil, M., Europhysics Letters 926, 69001 (2010).

[Netterfield 2002] Netterfield, C. B. et al., Astrophys. J. 571, 604 (2002).

[Nojiri \& Odintsov 2003] Nojiri, S., Odintsov, S. D., Astrophys. J. 565, 1 (2003).

[Nojiri \& Odintsov 2003] Nojiri, S., Odintsov, S. D., Physics Letters B 562, 147 (2003).

[Nojiri \& Odintsov 2001] Nojiri, S., Odintsov, S. D., International Journal of Modern Physics A 16, 3273 (2001).

[Nojiri et al. 2005] Nojiri, S., Odintsov, S. D., Tsujikawa, S., Phys. Rev. D 71, 063004 (2005).

[Padmanabhan 2002] Padmanabhan, T., Phys. Rev. D 66, 021301 (2002).

[Padmanabhan \& Choudhury 2002] Padmanabhan, T., Choudhury, T. R., Phys. Rev. D 66, 081301 (2002).

[Padmanabhan 2003] Padmanabhan, T., Phys. Rep. 380, 235 (2003).

[Peebles \& Ratra 2003] Peebles, P. J., Ratra, B., Reviews of Modern Physics 75, 559 (2003).

[Peiris et al. 2003] Peiris, H. V. et al., Astrophys. J. Suppl. Ser. 148, 213 (2003).

[Perlmutter et al. 1999] Perlmutter, S. et al., Astrophys. J. 517, 565 (1999).

[Piazza \& Tsujikawa 2004] Piazza, F., Tsujikawa, S., J. Cosmol. Astropart. Phys. 7, 4 (2004).

[Ratra \& Peebles 1988] Ratra, B., Peebles, P. J. E., Phys. Rev. D 37, 3406 (1988).

[Sadjadi \& Jamil 2011] Sadjadi, H. M., Jamil, M., General Relativity \& Gravitation 43, 1759 (2011).

[Sahni \& Shtanov 2003] Sahni, V., Shtanov, Y., J. Cosmol. Astropart. Phys. 11, 14 (2003).

[Seljak et al. 2006] Seljak, U., Slosar, A., McDonald, P., J. Cosmol. Astropart. Phys. 10, 14 (2006).

[Sen 2002a] Sen, A., JHEP 7, 65 (2002a). 
[Sen 2002b] Sen, A., JHEP 4, 48 (2002b).

[Sen 2002] Sen, A., Modern Physics Letters A 17, 1797 (2002).

[Sen 1999] Sen, A., JHEP 10, 8 (1999).

[Setare \& Jamil 2011] Setare, M. R., Jamil, M., General Relativity \& Gravitation 43, 293 (2011).

[Setare \& Jamil 2010a] Setare, M. R., Jamil, M., EPL (Europhysics Letters). 92, 49003 (2010).

[Setare 2007f] Setare, M., Physics Letters B 654, 1 (2007f).

[Setare 2007a] Setare, M. R., European Physical Journal C 52, 689 (2007a).

[Setare 2007b] Setare, M. R., Physics Letters B 653, 116 (2007b).

[Setare 2007c] Setare, M. R., Physics Letters B 648, 329 (2007c).

[Setare 2007d] Setare, M. R., Physics Letters B 644, 99 (2007d).

[Setare 2007e] Setare, M. R., European Physical Journal C 50, 991 (2007e).

[Setare 2006] Setare, M. R., Physics Letters B 642, 421 (2006).

[Setare \& Jamil 2010b] Setare, M. R., Jamil, M., Physics Letters B 690, 1 (2010b).

[Setare \& Jamil 2010c] Setare, M. R., Jamil, M., J. Cosmol. Astropart. Phys. 2, 10 (2010c).

[Setare \& Shafei 2006] Setare, M. R., Shafei, S., J. Cosmol. Astropart. Phys. 9, 11 (2006).

[Setare \& Vagenas 2008] Setare, M. R., Vagenas, E. C., Physics Letters B 666, 111 (2008).

[Shao et al. 2007] Shao, Y., Gui, Y.-X., Wang, W., Modern Physics Letters A 22, 1175 (2007).

[Shen et al. 2005] Shen, J., Wang, B., Abdalla, E., Su, R.-K., Physics Letters B 609, 200 (2005).

[Sheykhi \& Jamil 2011] Sheykhi, A., Jamil, M., General Relativity \& Gravitation 43, 2661 (2011).

[Sheykhi 2009] Sheykhi, A., Physics Letters B 681, 205 (2009).

[Sheykhi et al. 2010] Sheykhi, A. et al., (arXiv:1005.4541) [gen-ph].

[Sheykhi 2010] Sheykhi, A., Astrophys. J. 27, 025007 (2010).

[Sheykhi \& Jamil 2011] Sheykhi, A., Jamil, M., Physics Letters B 694, 284 (2011).

[Seievers 2003] Sievers, J. L. et al., Astrophys. J. 591, 599 (2003).

[Spergel 2007] Spergel, D. N. et al., Astrophys. J. Suppl. Ser. 170, 377 (2007).

[Spergel 2003] Seprgel, D. N. et al., Astrophys. J. Suppl. Ser. 148, 175 (2003).

[Tegmark et al. 2004] Tegmark, M. et al., Phys. Rev. D 69, 103501 (2004).

[t'Hooft 1993] 't Hooft, G.,(arXiv:gr-qc/9310026) [gr-qc].

[Wang et al. 2005a] Wang, B., Gong, Y., Su, R.-K., Physics Letters B 605, 9 (2005a).

[Wang et al. 2005b] Wang, B., Gong, Y., Abdalla, E., Astrophys. J. 624, 141 (2005b).

[Wang \& Xu 2010] Wang, Y., Xu, L., Phys. Rev. D 81, 083523 (2010). 
[Wei 2009] Wei, H., Commun. Theor. Phys. 52, 743 (2009).

[Wei \& Cai 2008] Wei, H., Cai, R.G., Physics Letters B 660, 113 (2008).

[Wei et al. 2010] Wei, S.W., Liu, Y.X., Wang, Y.Q., Guo, H., (arXiv:1002.1550). [hep-th].

[Weinberg 1989] Weinberg, S., Reviews of Modern Physics 61, 1 (1989).

[Wetterich 1988] Wetterich, C., Nuclear Physics B 302, 668 (1988).

[Zhang \& Zhu 2006] Zhang, H., Zhu, Z.H., Phys. Rev. D 73, 043518 (2006).

[Zhang 2009] Zhang, X., Phys. Rev. D 79, 103509 (2009).

[Zhang \& Wu 2007] Zhang, X., Wu, F.Q., Phys. Rev. D 76, 023502 (2007).

[Zhang 2006] Zhang, X., Phys. Rev. D 74, 103505 (2006).

[Zhang \& Wu 2005] Zhang, X., Wu, F.Q., Phys. Rev. D 72, 043524 (2005).

[Zimdahl 2003] Zimdahl, W., Pavón, D., General Relativity and Gravitation 35, 413 (2003).

[Zimdahl 2001] Zimdahl, W., Pavón, D., Chimento, L. P., Physics Letters B 521, 133 (2001).

[Zhu \& Ren 2009] Zhu, T., Ren, J.R., European Physical Journal C 62, 413 (2009). 